\title{
Applying the Background-Source separation algorithm to Chandra Deep Field South data
}

\author{
F. Guglielmetti ${ }^{1}$, H. Böhringer ${ }^{1}$, R. Fischer ${ }^{2}$, P. Rosati ${ }^{3}$, and P. Tozzi ${ }^{4}$
}

An analysis is performed to test the sensitivity and the internal consistency of the Background-Source separation (BSS) algorithm (see Ref. [2] and proceeding Guglielmetti et al. in this volume) with sources on real fields from pointed observations. The employed field is the Chandra Deep Field South (CDF-S) 2 Ms data [3]. The optimal energy band to detect the emission from both point-like and extended sources is between $0.5-2.0 \mathrm{keV}$. Therefore, this test is concentrated on this energy range. The main advantages of testing real data with respect to simulated ones reside on the fact that real data are characterized by a complex background, a complex point-spread function dependence across the field, source confusion and a wide range of source properties. These characteristics intrinsic to real observations are not easily elaborated with artificial data. Therefore, the CDF-S 2 Ms data are separated in four images of $500 \mathrm{ks}$ exposure time each.

The BSS algorithm is applied on each of the four images. The exponential prior probability density function of the source signal is chosen and 25 pivots equally spaced are used for the background rate estimation. Scales in the range value $0.5-13$ arcsec are used in the multiresolution analysis. A threshold value of $P_{\text {source }} \geq 0.9$ is chosen to separate false-positives in source detection from true sources. No contaminations due to steep changes in the exposure time map are seen both in the background map and in the source probability maps. The multiresolu-

Fabrizia Guglielmetti · Hans Böhringer

Max-Planck-Institut für extraterrestrische Physik, Giessenbachstrasse, D-85748 Garching, Germany, e-mail: fabrizia@mpe.mpg.de, hxb@mpe.mpg.de

Rainer Fischer

Max-Planck-Institute für Plasmaphysik, Boltzmannstrasse 2, D-85748 Garching, Germany, e-mail: Rainer.Fischer@ipp.mpg.de

Rosati Piero

European Southern Observatory, Karl-Schwarzschild-Strasse 2, D-85748 Garching, Germany, email: prosatideso.org

Paolo Tozzi

INAF-OATs, Via Tiepolo 11, I-34143 Trieste, Italy, e-mail: tozzi@oats. inaf.it 
tion analysis provides for the detection of a wide range of source fluxes and their complex morphologies.

The internal consistency of the BSS algorithm is tested comparing each CDF-S $500 \mathrm{ks}$ data in pairs. Source positions (right ascension and declination), fluxes and extent (i.e. the estimated size of the detected sources) are taken into account. The difference of position estimates are within $1 \sigma$ (Fig.1 a), while the ones of fluxes and extents are within $3 \sigma$ (Fig. $1 \mathbf{b}$ ). Although $70 \%$ of the sources in the CDF-S region are characterized by $X$-ray variability [4], Poisson fluctuations and contaminations by other sources in the fields can increase the uncertainties estimated for the source flux and extent measurements. The BSS estimates of source parameters are internally consistent.

A sensitivity analysis is performed on the four CDF-S $500 \mathrm{ks}$ data and the results are compared to published ones: CDF-S 1 Ms [1] and 2 Ms [3] data. The information about the sensitivity and the reliability of the survey are described by the sky coverage and the $\log N-\log S$ distribution. The estimated sky coverage and the $\log N$ $\log S$ distribution depend on the algorithm employed for source detection. The BSS background maps are used to construct the flux limit map of each estimated sky coverage. Hence, vignetting effects and background variations are already accounted in the coverage. The $\log N-\log S$ distributions are computed from the respective sky coverage. It results that the $\log N-\log S$ distributions obtained with the four CDF-S 500 ks data are in agreement with the published ones in Refs. [1, 3]: See Fig.1 c.

Applying the BSS algorithm to the CDF-S data, we prove that the BSS algorithm provides for a reliable detection of sources and estimation of source and background parameters. An extensive application of the technique is addressed in a forthcoming paper (Guglielmetti et al., in preparation).

\section{References}

1. Giacconi et al.: Chandra Deep Field South: The 1 Ms Catalog. ApJS 139, 369-410 (2002)

2. Guglielmetti, F., Fischer, R., Dose, V.: Background-source separation in astronomical images with Bayesian probability theory - I. The method. MNRAS 396, 165-190 (2009)

3. Luo, B. et al.: The Chandra Deep Field-South Survey: 2 Ms Source Catalogs. ApJS 179, 19-36 (2008)

4. Paolillo, M. et al.: Prevalence of X-Ray Variability in the Chandra Deep Field-South. ApJ 611, 93-106 (2004) 

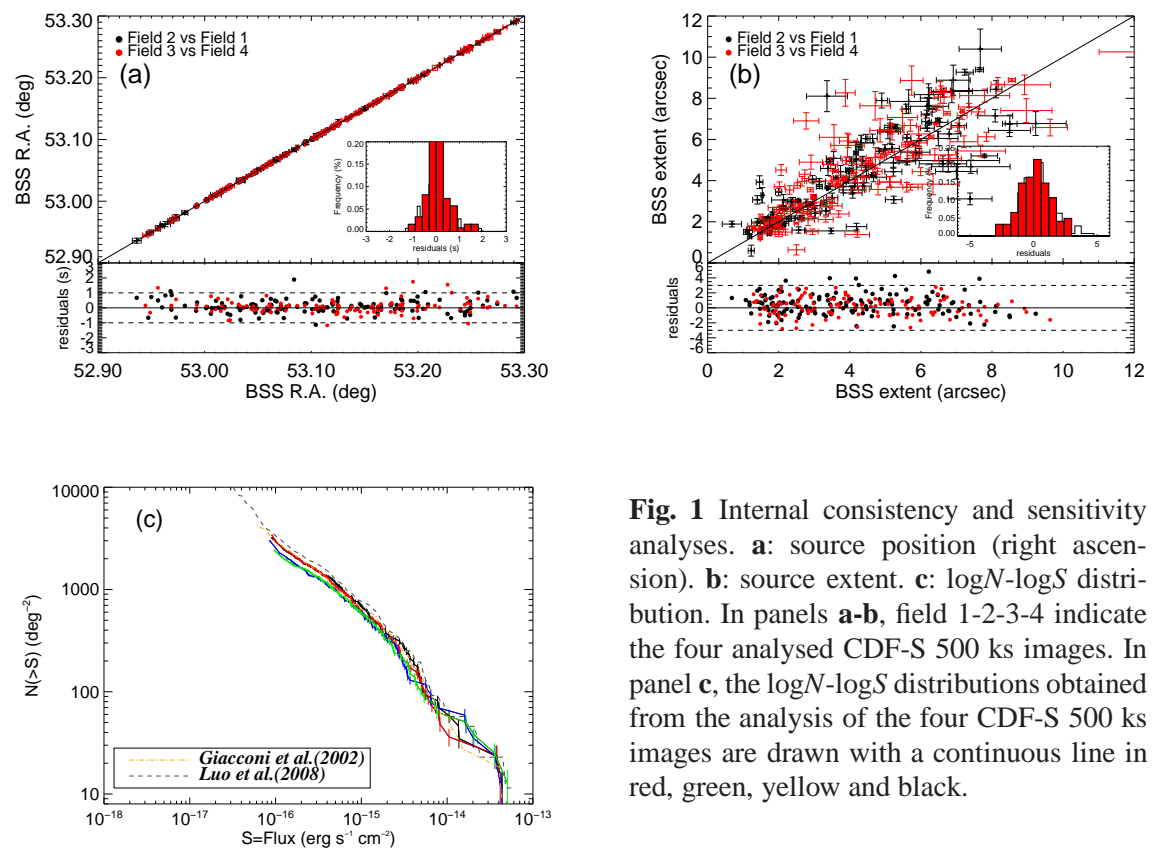

Fig. 1 Internal consistency and sensitivity analyses. a: source position (right ascension). b: source extent. c: $\log N-\log S$ distribution. In panels a-b, field 1-2-3-4 indicate the four analysed CDF-S $500 \mathrm{ks}$ images. In panel c, the $\log N-\log S$ distributions obtained from the analysis of the four CDF-S $500 \mathrm{ks}$ images are drawn with a continuous line in red, green, yellow and black. 